\title{
Editorial
}

\section{Vitamin $D$ in public health nutrition}

The year 2013 was again a very active year in the vitamin D field. The selection of papers discussed here focus on vitamin D intake, fortification, supplements, and vitamin D status and its associations with diseases and health. Here, we discuss several of the papers in the context of methodological challenges and gaps in current knowledge.

\section{Marker and cut-points}

Serum 25-hydroxyvitamin D concentration (25(OH)D) is considered the best marker of vitamin D status, with low concentrations indicating low status. However, cut-points for vitamin D deficiency and insufficiency vary, reflecting variable views among experts. Notably, the Institute of Medicine $^{(1)}$ concluded in 2010 that there are no systematic reviews on the cut-points, and this still stands. Papers by Chang $e t a l^{(2)}$ and Lee $e t ~ a l{ }^{(3)}$ used a cut-off of vitamin $\mathrm{D}<50 \mathrm{nmol} / 1$ to represent deficiency, reflective of the cut-offs recommended by the Institute of Medicine ${ }^{(1)}$, the Endocrine Society ${ }^{(4)}$ and the Nordic Nutrition Recommendations ${ }^{(5)}$. The paper by Oliveira et al. ${ }^{(6)}$ used a cut-off of $\leq 25 \mathrm{nmol} / 1$ to assess deficiency ${ }^{(7)}$ whereas Wallingford et $a l^{(8)}$ and Golbahar et al. ${ }^{(9)}$ reported vitamin D deficiency as serum $25(\mathrm{OH}) \mathrm{D}<30 \mathrm{nmol} / \mathrm{l}$ (risk for bone health) in line with the Institute of Medicine recommendations ${ }^{(1)}$. Other papers separated vitamin $\mathrm{D}$ status into quartiles ${ }^{(10)}$ and tertiles ${ }^{(11)}$, which may be valid for their research but hinders overall assessment of vitamin D status against established cut-offs.

\section{5-Hydroxyvitamin D assays}

Also problematic is that the methods used to assess $25(\mathrm{OH}) \mathrm{D}$ differ among the papers. It is well known that there is a large variation among methods and laboratories using the same methods ${ }^{(12-16)}$, leading to different estimates of vitamin $\mathrm{D}$ deficiency in a population ${ }^{(15)}$. The US National Institutes of Health recently started the Vitamin D Standardization Project (http://ods.od.nih.gov/Research/ VitaminD.aspx), which aims to harmonize and standardize 25(OH)D assessment - useful, for example, for national surveys ${ }^{(17,18)}$. A key factor in this process is the development of a 25(OH)D standard by the National Institute of Standards and Technology in the USA. Knowing that there is large variation in results from different assays, validating the assays used in individual studies is critical for comparing results across studies.

\section{Estimating vitamin D intake}

Estimating dietary intake of vitamin D is another challenge. In populations where natural vitamin $\mathrm{D}$ sources are few, food diaries are usually not enough to give a good estimation of vitamin D intake on the individual level and cannot be used in regression analyses. Moreover, many food composition databases do not cover all vitamin D sources. In an Irish study on children and teenagers ${ }^{(19)}$ food intake was recorded by either a $7 \mathrm{~d}$ weighed food diary (children) or a $7 \mathrm{~d}$ semi-weighed food diary (teens). Both food diary methods were based on the methodology used in the national North/South Ireland Food Consumption Survey ${ }^{(20)}$. Interestingly, they had to use British, Irish, American and Danish food composition databanks to cover all the important vitamin D sources for the study. This reflects a problem present in many countries - that food composition data may not represent real vitamin D intake.

\section{Influence of sun exposure}

Sun exposure is an important regulator of $25(\mathrm{OH}) \mathrm{D}$ concentration but also difficult to estimate. Vitamin D is produced in human skin when exposed to UVB irradiation, whether natural (sunshine) or artificial. But vitamin D production in human skin is affected by many external and individual factors. At latitudes above $\sim 50^{\circ} \mathrm{N}$, both the qualitative and quantitative properties of the sunlight are not sufficient for vitamin D production during part of the year ${ }^{(21)}$. The ozone layer also effectively absorbs UVB light, and clouds can attenuate UVB radiation by as much as $99 \%$. Surface reflection, especially from snow, can reflect up to $95 \%$ of UVB radiation. And at the individual level, time spent outdoors, sunscreen use and clothing affect sun-induced vitamin $\mathrm{D}$ production. Further, individuals with initially low $25(\mathrm{OH}) \mathrm{D}$ seem to have a lower threshold concentration for vitamin $\mathrm{D}$ production in skin compared with individuals with higher concentrations, and sun-induced vitamin $\mathrm{D}$ production can be up to six times higher in people with pale skin compared with people with dark $\operatorname{skin}^{(21)}$.

Despite the difficulty of estimating sun exposure, many instruments - questionnaires as well as devices for recording individual UV irradiation - have been developed to do so for research purposes. In this issue, Cook and co-workers ${ }^{(22)}$ describe a telephone-administered questionnaire used in the Ovarian Cancer in Alberta (OVAL) 
Study developed to assess lifetime exposure to vitamin D from sun-induced vitamin D synthesis and diet, based on assessment for every tenth year, in ninety females. The authors tested it against the questionnaire used in the Geraldton Skin Cancer Prevention Survey, considered a gold standard, in a cross-over design. The new OVAL questionnaire gave equivalent results to the Geraldton survey, with estimated average lifetime sun exposure reported in the two studies as $411 \mathrm{~h} /$ year and $358 \mathrm{~h} /$ year, respectively. The authors speculatively conclude that sun exposure may exceed the contribution of dietary intake to total vitamin D exposure during the warmer months of the year. In another study in this issue, Wallingford et al. ${ }^{(8)}$ also addressed the contribution of UV irradiation from sun and tanning beds in their study on predictors of vitamin D status in premenopausal women (see below).

\section{Predicting vitamin D status}

Papers by Golbahar et $a l^{(9)}$ and Wallingford et $a l^{(8)}$ in this issue discuss a range of predictors of vitamin D status. Wallingford et al. ${ }^{(8)}$ identified vitamin D supplement use, tanning bed use and season as predictors of vitamin D status in eighty-three premenopausal, shift-working nurses in Canada. Only $4 \%$ were deficient $(<30 \mathrm{nmol} / \mathrm{l})$ and $5 \%$ had insufficient $(<50 \mathrm{nmol} / \mathrm{l}) 25(\mathrm{OH}) \mathrm{D}$ concentrations after summer; the percentages after winter/spring were $6 \%$ and $7 \%$, respectively. In their study in 500 Bahrainis, Golbahar and co-authors ${ }^{(9)}$ identified female gender and age as risk factors for vitamin D deficiency (serum $25(\mathrm{OH}) \mathrm{D}<30 \mathrm{nmol} / \mathrm{l}$ ) along with season and conservative clothing style. Only season predicted vitamin D insufficiency (serum $25(\mathrm{OHD}<50 \mathrm{nmol} / \mathrm{l}$ ) in both males and females. Interestingly, summer months were associated with lower vitamin D status, possibly a result of high humidity and temperatures and reduced outside activities. The lower vitamin D status observed in women was attributed partly to women spending more time indoors and clothing style.

The high cost of screening for vitamin D insufficiency has spurred the development of questionnaires to predict the need for vitamin D assessment. Nabak et al. ${ }^{(23)}$ report high sensitivity $(\geq 89 \%)$ but low specificity $(\leq 35 \%)$ of predicting low or high risk for vitamin $\mathrm{D}$ insufficiency (serum $25(\mathrm{OH}) \mathrm{D}<50 \mathrm{nmol} / \mathrm{l}$ ) based on a composite score in postmenopausal women. Predictors incorporated in the score included race, skin type, BMI, sunscreen use and use of vitamin D supplements - predictors previously reported to be associated with vitamin D status. The authors concede that revision of the questionnaire to include questions relating to exercise and fatty fish intake might improve the performance of the questionnaire. The idea of being able to accurately predict who may or may not need vitamin D status screening has important financial implications. However, as already indicated, development of a questionnaire relies on known predictors of vitamin D. Furthermore, this work needs to be extended to men and to a wider variety of age ranges.

\section{Vitamin D status in children and adolescents}

Six of the papers in this issue focus on children and adolescents $^{(2,3,6,11,19,24)}$. Black and co-workers ${ }^{(19)}$ studied vitamin $\mathrm{D}$ intakes in a group of children and a group of teens in Ireland who had participated in the Children's and Teens' National Nutrition Surveys in 2004 and 2007. The results revealed that vitamin D intakes from food and fortified foods were very low in all age groups. Those taking supplements had a somewhat higher intake but did not reach the US Institute of Medicine dietary reference intake ${ }^{(1)}$. These results probably reflect the situation in many countries. Natural sources are few, and fortification may not be targeted to the right segment of the population. Fortification has to be carefully planned and researched to reach to the right individuals but also to minimize risk with excessive intake. Long-term supplementation is an option but, as seen in Black et al.'s study, may not be feasible on a population level in these age groups.

Supplements may be an important source of vitamin D in infants all over the world. Vitamin D supplements have been recommended to children aged 0-2 years in Northern Europe for decades to combat rickets ${ }^{(5)}$. Lehtonen et al. ${ }^{(24)}$ conducted a survey on the use of supplements in 2159 infants aged 1-6 months in Europe, North America and Australia based on data from the TRIGR study - a longitudinal study examining the effect of infant weaning on to a hydrolysed infant formula on incidence of type I diabetes in those with increased genetic susceptibility. Their results revealed that the majority of the infants were supplemented in Europe, approximately half in Canada, yet very few in the USA and Australia. Interestingly, longer maternal education, higher gestational age and older maternal age were studywide associated with greater use of supplements.

Three studies focus on associations between vitamin D status and health outcomes in children and/or adolescents from different parts of the world. In 160 Brazilian adolescents, of whom about 50\% were overweight, vitamin D intake was assessed by a $3 \mathrm{~d}$ food record. Vitamin D intake was low and no correlation between intake and $25(\mathrm{OH}) \mathrm{D}$ was apparent, which the authors suggest could be due to the food composition databank $^{(6)}$. However, seasonal variation was not taken into account, three days may be too short for assessing intake and the 25(OH)D assay was not specified. The authors also reported negative associations with weight excess, abdominal obesity, hypercholesterolaemia, higher serum parathyroid hormone concentration, insulin resistance, hyperinsulinaemia and hypertension. However, causality cannot be established and all outcomes could also be 
related to, for example, physical activity (related to time spent outside), although it was not associated with $25(\mathrm{OH}) \mathrm{D}$ concentration.

A study by Chung et $a l .{ }^{(11)}$ reported that three-quarters of a representative adolescent population ( $n$ 1466, age 10-19 years) enrolled in the Korea National Health and Nutrition Examination Survey (KHANES, 2009-2010) were vitamin D deficient. Vitamin D status in these participants was significantly related to markers of adiposity, fat mass percentage, insulin and impaired fasting glucose after adjustment for age, sex, season and physical activity. However, UVB exposure was not assessed and there was a lack of information pertaining to sunscreen use, visceral adiposity and total vitamin D intake - all reported predictors of vitamin D status. Lee et $a l^{(3)}$ also reported data on 1510 adolescents (age 12-18 years) enrolled in the 2008-2009 KHANES study. Independent predictors of low vitamin D status were winter season, higher education and a lack of supplementation with vitamin D. Both papers $^{(3,11)}$ appeared to use a cut-off value of $50 \mathrm{nmol} / \mathrm{l}$ as representative of vitamin D deficiency. In another study in a paediatric population, Chang et $a l^{(2)}$ reported prevalences of deficiency $(<50 \mathrm{nmol} / \mathrm{l})$ and insufficiency $(<75 \mathrm{nmol} / 1)$ of $21 \cdot 0 \%$ and $27.4 \%$ respectively in 1218 children from the Chinese province of Chengdu, Sichuan, displaying a lower level of deficiency when compared with the KHANES study. In that population vitamin D insufficiency and deficiency increased with age, and the association of vitamin D deficiency with elevated serum $\mathrm{Pb}$ concentrations was also reported. However, dietary intakes of essential nutrients known to influence vitamin D status were not investigated and collection of samples was not controlled based on seasonality.

\section{Vitamin $D$ and disease risk}

The association of vitamin D (insufficiency) with a variety of disease states/markers of disease in adults is well documented. In a cross-sectional study in 42730 participants (age 40-75 years) of the Health Professionals Follow-Up Study, an association between predictors of vitamin D and lower incidence of tooth loss and periodontitis was observed $^{(25)}$. The $25(\mathrm{OH}) \mathrm{D}$ score used in that study was updated for each follow-up, conducted biannually since 1988, for most of the components in the score, e.g. physical activity, lifestyle behaviour, physical characteristics. Dietary data were collected every 4 years by FFQ. The highest quintile of the updated predicted vitamin D score was associated with a $20 \%$ lower incidence of tooth loss compared with the lowest. Although the study cannot prove causality, the longitudinal study design with 20-year follow-up and large sample size are strengths. In a cross-sectional study in 6350 middle-aged and elderly participants in Norway ${ }^{(10)}$, no association was observed between serum 25(OH)D and symptoms of respiratory tract infection, adding complexity to this issue. Previous studies showed mixed results, and a systematic review has called for more rigorously designed trials to clarify the relationship between 'vitamin D and the immune response to infection, ${ }^{,(26)}$.

\section{Conclusion}

In conclusion, the studies discussed in this editorial contribute to the consensus that vitamin D deficiency and insufficiency are prevalent and not limited to a particular age group or geographical location. Fortification of foods and supplementation with vitamin D appear to improve status but rely on the consumption of these products by those at most risk, as well as on their availability and affordability. A number of well-established predictors of status, including gender, race, sunscreen use and UVB exposure, have been incorporated into questionnaires that may be able to predict those at risk of vitamin D deficiency. But what does remain uncertain is any clearly established risk of a low vitamin D status with respect to adverse health outcomes other than those related to bone health. Associations between vitamin D status and some markers of poor health were discussed but, as with most associations, randomized controlled trials are needed to confirm these associations. Fortunately, many large trials are currently ongoing in Europe and the USA. A recent systematic review has raised the possibility that low vitamin D status is a result of ill health rather than a cause ${ }^{(27)}$. In probably the largest systematic review of its type on vitamin D and ill health, the authors concluded that vitamin D intervention studies 'did not show an effect of vitamin D supplementation on disease occurrence', again raising the question of whether low vitamin D status is the cause or result of ill health. This latest systematic review and the findings emanating from the thirteen papers in this issue, described here, further articulate the urgent need for quality randomized controlled trials to be undertaken to delineate non-bone related health effects of a low vitamin D status. Furthermore, clarity and transparency are required when describing levels of vitamin $\mathrm{D}$ deficiency in published research and standardization of the use of cut-offs and assessment of vitamin D status will assist public health researchers in the design and execution of future studies.

Maxine P Bonham Christel Lamberg-Allardt Deputy Editors

\section{References}

1. Institute of Medicine Committee to Review Dietary Reference Intakes for Vitamin D and Calcium (2011) Dietary Reference Intakes for Calcium and Vitamin D [AC Ross, CL Taylor, AL Yatkine et al., editors]. Washington, DC: National Academies Press. 
2. Chang L, Liu X, Shi H et al. (2014) Association of 25hydroxyvitamin $\mathrm{D}$ with $\mathrm{Hb}$ and lead in children: a Chinese population-based study. Public Health Nutr 17, 827-832.

3. Lee YA, Kim HY, Hong H et al. (2014) Risk factors for low vitamin D status in Korean adolescents: the Korea National Health and Nutrition Examination Survey (KNHANES) 2008-2009. Public Health Nutr 17, 764-771.

4. Holick MF, Binkley NC, Bischoff-Ferrari HA et al. (2011) Evaluation, treatment, and prevention of vitamin D deficiency: an Endocrine Society clinical practice guideline. J Clin Endocrinol Metab 96, 1911-1930.

5. Lamberg-Allardt C, Brustad M, Meyer HE et al. (2013) Vitamin D - a systematic literature review for the 5th edition of the Nordic Nutrition Recommendations. Food Nutr Res 2013, 57.

6. Oliveira RM, Novaes JF, Azeredo LM et al. (2014) Association of vitamin D insufficiency with adiposity and metabolic disorders in Brazilian adolescents. Public Health Nutr 17, 787-794.

7. Bischoff-Ferrari HA, Giovannucci E, Willett WC et al. (2006) Estimation of optimal serum concentrations of 25-hydroxyvitamin D for multiple health outcomes. Am J Clin Nutr 84, 18-28.

8. Wallingford SC, Jones G, Kobayashi LC et al. (2014) UV and dietary predictors of serum 25-hydroxyvitamin D concentrations among young shift-working nurses and implications for bone density and skin cancer. Public Health Nutr 17, 772-779.

9. Golbahar J, Al-Saffar N, Altayab Diab D et al. (2014) Predictors of vitamin D deficiency and insufficiency in adult Bahrainis: a cross-sectional study. Public Health Nutr 17, 732-738.

10. Robertsen S, Grimnes G \& Melbye H (2014) Association between serum 25-hydroxyvitamin $\mathrm{D}$ concentration and symptoms of respiratory tract infection in a Norwegian population: the Tromsø Study. Public Health Nutr 17, 780-786.

11. Chung SJ, Lee YA, Hong H et al. (2014) Inverse relationship between vitamin $\mathrm{D}$ status and insulin resistance and the risk of impaired fasting glucose in Korean children and adolescents: the Korean National Health and Nutrition Examination Survey (KNHANES) 2009-2010. Public Health Nutr 17, 795-802.

12. Barake M, Daher RT, Salti I et al. (2012) 25-Hydroxyvitamin $\mathrm{D}$ assay variations and impact on clinical decision making. J Clin Endocrinol Metab 97, 835-843.

13. Bedner M, Lippa KA \& Tai SS (2013) An assessment of 25-hydroxyvitamin D measurements in comparability studies conducted by the Vitamin D Metabolites Quality Assurance Program. Clin Chim Acta 426, 6-11.
14. Carter GD, Berry JL, Gunter E et al. (2010) Proficiency testing of 25-hydroxyvitamin D (25-OHD) assays. J Steroid Biochem Mol Biol 121, 176-179.

15. Snellman G, Melhus H, Gedeborg R et al. (2010) Determining vitamin D status: a comparison between commercially available assays. PloS ONE 5, e11555.

16. Wallace AM, Gibson S, de la Hunty A et al. (2010) Measurement of 25-hydroxyvitamin D in the clinical laboratory: current procedures, performance characteristics and limitations. Steroids 75, 477-488.

17. Cashman KD, Kiely M, Kinsella M et al. (2013) Evaluation of Vitamin D Standardization Program protocols for standardizing serum 25-hydroxyvitamin D data: a case study of the program's potential for national nutrition and health surveys. Am J Clin Nutr 97, 1235-1242.

18. Sempos CT, Vesper HW, Phinney KW et al. (2012) Vitamin D status as an international issue: national surveys and the problem of standardization. Scand J Clin Lab Invest Suppl 243, 32-40.

19. Black LJ, Walton J, Flynn A et al. (2014) Adequacy of vitamin $\mathrm{D}$ intakes in children and teenagers from the base diet, fortified foods and supplements. Public Health Nutr 17, 721-731.

20. Harrington KE, Robson PJ, Kiely M et al. (2001) The North/ South Ireland Food Consumption Survey: survey design and methodology. Public Health Nutr 4, 1037-1042.

21. Engelsen O (2010) The relationship between ultraviolet radiation exposure and vitamin D status. Nutrients 2 , 482-495.

22. Cook LS, Moon BL, Dong Y et al. (2014) Reliability of selfreported sun exposure in Canadian women and estimation of lifetime exposure to vitamin D from sun and diet. Public Health Nutr 17, 747-755.

23. Nabak AC, Johnson RE, Keuler NS et al. (2014) Can a questionnaire predict vitamin D status in postmenopausal women? Public Health Nutr 17, 739-746.

24. Lehtonen E, Ormisson A, Nucci A et al. (2014) Use of vitamin D supplements during infancy in an international feeding trial. Public Health Nutr 17, 810-822.

25. Jimenez M, Giovannucci E, Krall Kaye E et al. (2014) Predicted vitamin D status and incidence of tooth loss and periodontitis. Public Health Nutr 17, 844-852.

26. Yamshchikov AV, Desai NS, Blumberg HM et al. (2009) Vitamin D for treatment and prevention of infectious diseases: a systematic review of randomized controlled trials. Endocr Pract 15, 438-449.

27. Autier P, Boniol M, Pizot C et al. (2014) Vitamin D status and ill health: a systematic review. Lancet Diabetes Endocrinol 2, 76-89. 